Article

\title{
In-Vitro Evaluation of Different Antimicrobial Combinations with and without Colistin Against Carbapenem-Resistant Acinetobacter Baumannii
}

\author{
Alessandra Oliva ${ }^{1, *(\mathbb{D}}$, Stefania Garzoli ${ }^{2}{ }^{\circ}$, Massimiliano De Angelis ${ }^{1}$, Carolina Marzuillo ${ }^{1}$, \\ Vincenzo Vullo ${ }^{1}$, Claudio M. Mastroianni ${ }^{1}$ and Rino Ragno ${ }^{3,4, *(D)}$ \\ 1 Department of Public Health and Infectious Diseases, Sapienza University, P.le Aldo Moro 5, \\ 00185 Rome, Italy; massimiliano.deangelis@uniroma1.it (M.D.A.) carolina.marzuillo@uniroma1.it (C.M.); \\ vincenzo.vullo@uniroma1.it (V.V.); claudio.mastroianni@uniroma1.it (C.M.M.) \\ 2 Department of Drug Chemistry and Technology, Sapienza University, P.le Aldo Moro 5, 00185 Rome, Italy; \\ stefania.garzoli@uniroma1.it \\ 3 Rome Center for Molecular Design, Sapienza University, P.le Aldo Moro 5, 00185 Rome, Italy \\ 4 Alchemical Dynamics s.r.l., 00125 Rome, Italy \\ * Correspondence: alessandra.oliva@uniroma1.it (A.O.); rino.ragno@uniroma1.it (R.R.); \\ Tel.: +39-0649970880 (A.O.); +39-0649913937 (R.R.); Fax: +390649972625 (A.O.); +39-0649913627 (R.R.)
}

Academic Editor: Francesc Rabanal Anglada

Received: 31 December 2018; Accepted: 27 February 2019; Published: 3 March 2019

\begin{abstract}
Carbapenem-resistant Acinetobacter baumannii (CR-Ab) infections are associated with high morbidity and mortality. The aim of the study was to evaluate the in-vitro activity of different antimicrobial combinations (with and without colistin, COL) against clinical isolates of $\mathrm{CR}-\mathrm{Ab}$ collected from patients with CR-Ab infection, including unconventional combinations such as COL + VANcomycin (VAN) and COL + rifampin (RIF). CR-Ab strains were collected from hospitalized patients at Sapienza University of Rome. Antimicrobial susceptibility patterns were determined throughout MIC50/90s whereas the synergistic activity was evaluated by qualitative (i.e., checkerboard) and quantitative (i.e., killing studies) methods. All the strains were found oxacillinase (OXA) producers and tigecycline (TIG) sensitive whereas 2 strains were resistant to COL. Application of the checkerboard method indicated complete synergism in COL combinations at different extension: $21.4 \%, 57.1 \%, 42.8 \%, 35.7 \%$ for COL + meropenem (MEM), COL + RIF, COL + VAN and COL + TIG, respectively, with the non-conventional combinations COL + VAN and COL + RIF exhibiting the highest rate of synergism. Regarding COL-free combination, complete synergism was observed in $35.7 \%$ of the strains for MEM + TIG. Killing studies showed that the combinations COL + MEM, COL + TIG and MEM + TIG were bactericidal and synergistic against both colistin-sensitive and low colistin-resistant strains whereas only the combinations COL + VAN and COL + RIF showed an early and durable bactericidal activity against all the tested strains, with absence of growth at $24 \mathrm{~h}$. This study demonstrated that COL-based combinations lead to a high level of synergic and bactericidal activity, especially COL + VAN and COL + RIF, even in the presence of high level of COL resistance.
\end{abstract}

Keywords: carbapenem-resistant $A$. baumannii; colistin; vancomycin; antimicrobial combinations; synergy; time-kill studies

\section{Introduction}

The rapid spread of multidrug-resistant bacteria (MDR) such as carbapenem-resistant (CR) Enterobacteriaceae (CR-E), CR Acinetobacter baumannii (CR-Ab) and MDR Pseudomonas aeruginosa (MDR-Pa) has become a public health concern, especially in some countries where the diffusion of carbapenem-resistant microorganisms is nowadays endemic [1,2]. In particular, CR-Ab represents a 
major challenge for physicians due to the high mortality rates especially in the ICU and its capability to persist in the environment [3-5].

Therapeutic options are severely limited $[2,6]$ and are mainly based on colistin-containing combinations. However, the ability of $\mathrm{CR}-\mathrm{Ab}$ to acquire resistance even to colistin (COL) further limits, if not completely precludes, the therapeutic choices. In this scary scenario, the lack of activity of new antimicrobials nowadays available in many countries imposes new efforts in finding the best therapeutic approach against this pathogen [7]. Furthermore, although in the pipeline there are several promising agents with activity towards all $\mathrm{CR}$ microorganisms including $\mathrm{CR}-\mathrm{Ab}$ (i.e., cefiderocol), they are still in in early clinical development of and will be eventually available only in the coming years [8].

Polymyxins at the moment are the antimicrobials with the greatest level of in-vitro activity against $\mathrm{CR}-\mathrm{Ab}$ [9]. In the studies evaluating the synergistic activity of COL-containing combinations, results suggested that COL plus carbapenems or COL plus antimicrobials active only against Gram-positive bacteria, such as vancomycin (VAN) and rifampin (RIF) can be considered the most active in-vitro combinations [10-12].

However, the potential survival benefit obtained with combination therapy instead of monotherapy in infections caused by CR-Ab is still debated, following a recent randomized clinical trial resulted with no advantages of COL combined with meropenem (MEM) versus COL monotherapy for severe $\mathrm{CR}-\mathrm{Ab}$ infections [13]. On the other hand, the use of COL-based combination (i.e., COL plus RIF) gave a higher rate of microbiological eradication compared with that obtained using monotherapy [14], furthermore, the most likely possibility of developing resistance during COL monotherapy should be taken into account, possibly contributing to the rationale for combination use in CR-Ab infections.

Apart from resistance, which has been increasingly reported in the last years [15], the use of COL in the clinical practice might be limited by its toxic effect; therefore, developing alternative and effective COL-free combinations are of prime importance [16].

Based on the above mentioned studies, the aim of the research reported herein was to evaluate the in-vitro activity of antimicrobial combinations with and without COL against clinical strains of CR-Ab with different clonal types, with particular emphasis on the non-conventional regimens containing COL plus VAN or RIF. In addition, clinical and demographic data of patients were evaluated.

\section{Results}

\subsection{Bacterial Strains and Clinical Data Collection}

Overall, 14 patients with CR-Ab infection (3 bloodstream infections; 2 skin and soft tissue infection; 9 hospital-acquired pneumonia) were included in the study. Clinical characteristics and outcomes of patients are shown in Table 1. All the patients received colistin-based combinations [10: COL + high dosage (i.e., 6 grams/d) of MEM; 2: COL +TIG; 2: COL + RIF], with a total of 6/14 (43\%) also receiving VAN. The overall clinical cure was $12 / 14(85.7 \%)$. CR-Ab strains were isolated from upper/lower respiratory tract fluids $(n=9)$, blood $(n=3)$ and purulent drainages $(n=2)$.

\subsection{Molecular Typing by Pulsed-Field Gel Electrophoresis}

Pulsed-field gel electrophoresis (PFGE) analysis was reliable in 13 out of the $14 \mathrm{CR}-\mathrm{Ab}$ isolated strains. Overall, 5 clones were detected: A, B, C, D, E (Figure 1). Among these, clone B was the most common $(n=4)$ and was found exclusively in the SUR Paediatric Intensive Care Unit (PICU) whereas clone $\mathrm{C}$ was detected in only one strain isolated from a patient hospitalized in a different Intensive Care Unit before being transferred to SUR hospital. Clones A, D and E were found in isolated patients hospitalized in different wards of the hospital (i.e., Internal Medicine, Gerontology and Pneumology). 
Table 1. Clinical characteristics of patients $(n=14)$ with CR-Ab infection.

\begin{tabular}{ccccc}
\hline Patient & Type of Infection & Therapy & Duration of Therapy (days) & Outcome \\
\hline 1 & BSI & $\mathrm{C}+\mathrm{M}+\mathrm{V}$ & 14 & Cured \\
2 & $\mathrm{HAP}$ & $\mathrm{C}+\mathrm{M}+\mathrm{V}$ & 13 & Cured \\
3 & $\mathrm{SSI}$ & $\mathrm{C}+\mathrm{T}+\mathrm{V}$ & 30 & Cured \\
4 & $\mathrm{SSI}$ & $\mathrm{C}+\mathrm{R}$ & 14 & Cured \\
5 & $\mathrm{BSI}$ & $\mathrm{C}+\mathrm{M}$ & 30 & Cured \\
6 & $\mathrm{HAP}$ & $\mathrm{C}+\mathrm{V}+\mathrm{R}$ & 14 & Cured \\
7 & $\mathrm{BSI}$ & $\mathrm{C}+\mathrm{M}+\mathrm{V}$ & 6 & Died \\
8 & $\mathrm{HAP}$ & $\mathrm{C}+\mathrm{M}$ & 17 & Cured \\
9 & $\mathrm{HAP}$ & $\mathrm{C}+\mathrm{M}$ & 14 & Cured \\
10 & $\mathrm{HAP}$ & $\mathrm{C}+\mathrm{M}$ & 14 & Cured \\
11 & $\mathrm{HAP}$ & $\mathrm{C}+\mathrm{M}$ & 14 & Cured \\
12 & $\mathrm{HAP}$ & $\mathrm{C}+\mathrm{T}$ & 14 & Cured \\
13 & $\mathrm{HAP}$ & $\mathrm{C}+\mathrm{M}$ & 10 & Died* \\
14 & HAP & $\mathrm{C}+\mathrm{M}+\mathrm{V}$ & 14 & Cured \\
\hline
\end{tabular}

BSI: Bloodstream infection. SSI: Skin and Soft Tissue Infection. HAP: Hospital-acquired pneumonia. *: pt\#7 died because of CR-Ab infection; pt\#13 died for reason other than CR-Ab infection. C: colistin; M: meropenem; V: vancomycin; R: rifampin; T: tigecycline.

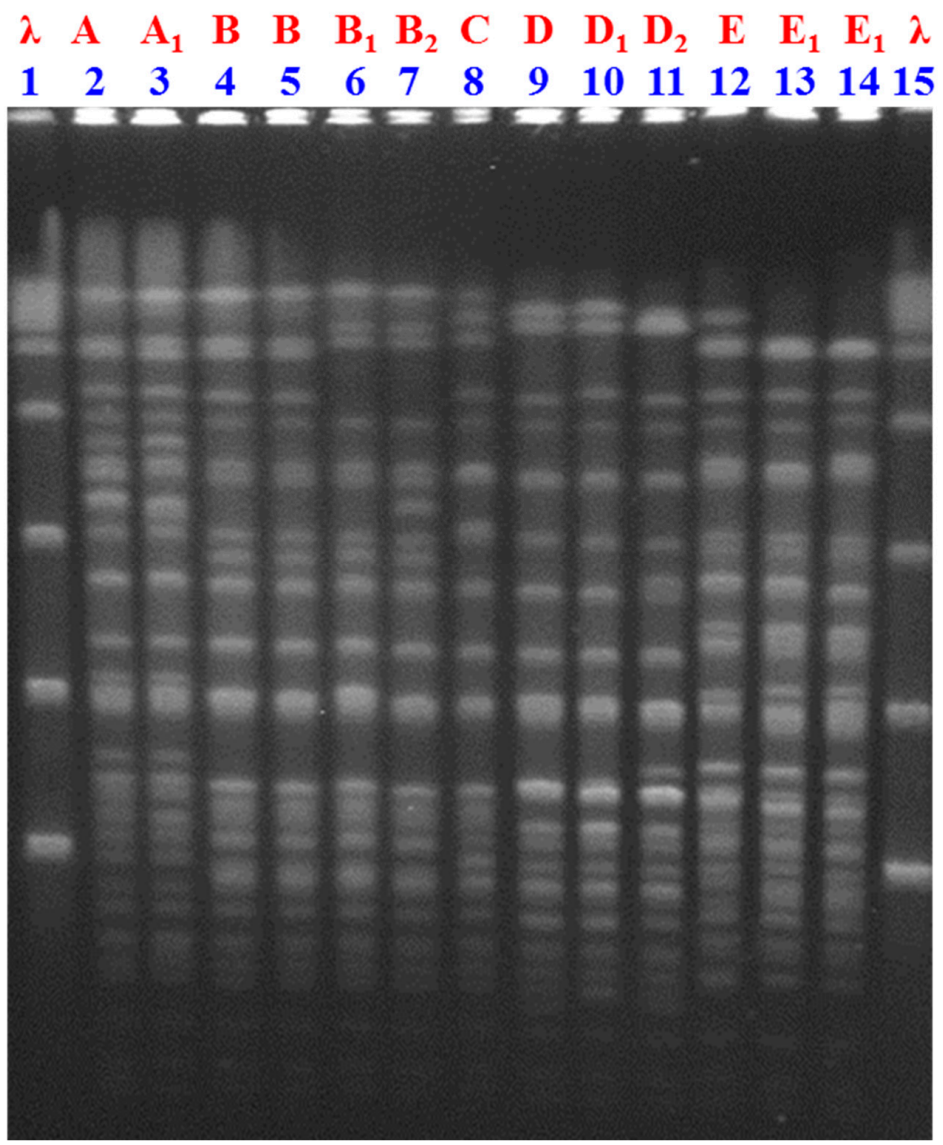

Figure 1. ApaI pulse-field gel electrophoresis (PFGE) patterns of $A$ baumannii. Lanes 1, 15 contain molecular size patterns (lambda ladder). Lanes 2,3 (PFGE pattern A), 4-7 (B), 8 (C), 9-11 (D) and 12-14 (E). Isolates with more than 3 fragment variations were assumed to represent strains with major patterns (assignment of capital letters), while isolates with 1-3 fragment differences were considered subtypes (capital letters with numerical subscripts).

\subsection{Antimicrobial Activity}

Throughout the VITEK-2 system, all the strains were carbapenem-resistant (MIC > 16mg/L) whereas Table 2 summarizes the in-vitro susceptibility of CR-Ab throughout MBD and E-test methods. Throughout MBD, MICs50/90 were 128/256 mg/L for MEM, 64/128 mg/L for VAN, 256/512 mg/L 
for GEN, 0.5/1 mg/L for TIG, 0.5/2 mg/L for COL and 16/512 mg/L for RIF. In particular, 2 strains were resistant to COL [1 with low-level of resistance (COL-LR, MIC $4 \mathrm{mg} / \mathrm{L}$ ), 1 with high-level of resistance (COL-HR, MIC $256 \mathrm{mg} / \mathrm{L}$ )]. All the strains were oxacillinase (OXA) producers.

Table 2. In-vitro susceptibility of carbapenem-resistant Acinetobacter baumannii (CR-Ab) throughout macrobroth dilution (MBD) and E-test methods.

\begin{tabular}{cccccccccc}
\hline & Antibiotic & MEM & \multicolumn{2}{c}{ COL } & VAN & RIF & GEN & \multicolumn{2}{c}{ TIG } \\
\cline { 2 - 9 } & Method & MBD & MBD & E-Test & MBD & MBD & MBD & E-Test & MBD \\
\hline & 1 & 128 & 1.000 & 0.750 & 256 & 8 & 4 & 2.00 & 0.50 \\
& 2 & 256 & 0.250 & 0.300 & 32 & 4 & $>512$ & 1.50 & 1.00 \\
& 3 & 256 & 0.060 & 0.380 & 64 & $>512$ & 2 & 0.75 & 1.00 \\
Patients/ & 4 & 128 & 0.032 & 0.075 & 128 & $>512$ & $>512$ & 2.00 & 0.50 \\
Clinical & 5 & 32 & 0.125 & 0.380 & 64 & 4 & $>512$ & 1.00 & 1.00 \\
Isolates & 7 & 128 & 4.000 & 4.00 & 64 & 4 & 512 & 3.00 & 0.50 \\
& 8 & 512 & 0.015 & 0.750 & 128 & $>512$ & $>512$ & 2.00 & 1.00 \\
& 15 & 256 & 0.030 & 0.380 & 128 & 4 & 32 & 1.50 & 0.25 \\
& 10 & 32 & 2.000 & 1.000 & 128 & 2 & $>512$ & 1.50 & 0.50 \\
& 11 & 128 & 0.500 & 1.500 & 64 & 16 & 64 & 0.75 & 0.75 \\
& 12 & 8 & 256.0 & 128.0 & 32 & $>512$ & 2 & 1.00 & 0.25 \\
& 13 & 64 & 0.125 & 0.500 & 32 & 4 & $>512$ & 1.50 & 0.25 \\
& 14 & 32 & 0.500 & 0.500 & 64 & 4 & 256 & 2.00 & 0.50 \\
\hline
\end{tabular}

MEM: meropenem; COL: colistin; VAN: vancomycin; RIF: rifampin; GEN: gentamicin; TIG: tigecycline.

Complete synergism was observed in 3 out of $14(21.4 \%), 8$ out of $14(57.1 \%), 6$ out of $14(42.8 \%)$ and 5 out of $14(35.7 \%)$ strains using combinations COL + MEM, COL + RIF, COL + VAN and COL + TIG, respectively. Among COL-free combinations, MEM + TIG showed complete synergism in 5/14 $(35.7 \%)$ (Table 3).

Table 3. Synergism of different combinations against CR-Ab throughout checkerboard method. The 14 clinical isolated as listed in Table 1 were used.

\begin{tabular}{|c|c|c|c|c|c|c|c|c|c|c|c|}
\hline \multirow{2}{*}{ Strains } & \multirow{2}{*}{$\begin{array}{c}\text { PFGE } \\
\text { Pattern }\end{array}$} & \multicolumn{2}{|c|}{ COL + MEM } & \multicolumn{2}{|c|}{$\mathrm{COL}+\mathrm{RIF}$} & \multicolumn{2}{|c|}{$\mathrm{COL}+\mathrm{VAN}$} & \multicolumn{2}{|c|}{$\mathrm{COL}+\mathrm{TIG}$} & \multicolumn{2}{|c|}{ MEM + TIG } \\
\hline & & FICI $^{\circ}$ & Syn & FICI & Syn & FICI & Syn & FICI & Syn & FICI & Syn \\
\hline 1 & B & 0.375 & S & 0.375 & S & 0.250 & S & 0.375 & S & 1.000 & I \\
\hline 2 & $\mathrm{~B}_{1}$ & 0.625 & I & 0.375 & $S$ & 0.625 & I & 1.000 & I & 0.500 & $S$ \\
\hline 3 & B & 0.625 & I & 0.625 & I & 0.750 & I & 0.625 & I & 0.375 & $S$ \\
\hline 4 & A & 0.750 & I & 0.625 & I & 0.625 & I & 1.000 & I & 0.750 & I \\
\hline 5 & $\mathrm{D}$ & 0.625 & I & 0.500 & S & 0.250 & $S$ & 0.250 & S & 0.625 & I \\
\hline $6^{*}$ & $\mathrm{C}$ & 0.250 & S & 0.250 & $S$ & $0.250^{\circ}$ & $S$ & 0.250 & S & 0.375 & $S$ \\
\hline 7 & $\mathrm{~A}_{1}$ & 1.000 & I & 1.000 & I & 1.000 & I & 2.000 & I & 0.625 & I \\
\hline 8 & $\mathrm{E}_{1}$ & 0.625 & I & 0.750 & I & 0.375 & $S$ & 2.000 & I & 1.000 & I \\
\hline 9 & $\mathrm{~B}_{2}$ & 0.750 & I & 0.750 & I & 0.625 & I & 2.000 & I & 0.250 & $S$ \\
\hline 10 & $\mathrm{E}$ & 0.250 & $S$ & 0.250 & $S$ & 0.250 & $S$ & 0.250 & $S$ & 0.625 & I \\
\hline 11 & - & 1.000 & I & 0.250 & S & 0.375 & $S$ & 0.375 & S & 0.375 & $S$ \\
\hline $12^{* *}$ & $\mathrm{D}_{2}$ & 0.750 & I & 0.250 & $S$ & $0.625 \S$ & I & 2.000 & I & 1.000 & I \\
\hline 13 & $\mathrm{D}_{1}$ & 2.000 & I & 0.625 & I & 0.625 & I & 2.000 & I & 0.625 & I \\
\hline 14 & $\mathrm{E}_{1}$ & 0.625 & I & 0.250 & S & 0.625 & I & 0.750 & I & 0.625 & I \\
\hline Total n & & & $3 / 14$ & & $8 / 14$ & & $6 / 14$ & & $5 / 14$ & & $5 / 14$ \\
\hline Total (\%) & & & 21.4 & & 57.1 & & 42.8 & & 35.7 & & 35.7 \\
\hline
\end{tabular}

*: strain with low-level of COL-resistance (COL-LR, MIC $4 \mathrm{mg} / \mathrm{L}$ ); ${ }^{* *}$ : strain with high-level of COL-resistance (COL-HR, MIC $256 \mathrm{mg} / \mathrm{L}$ ); ${ }^{\circ}$ : FICI 0.250 was obtained at the following concentrations: $0.5 \mathrm{mcg} / \mathrm{mL}$ COL $(0.125 \times \mathrm{MIC})+8 \mathrm{mcg} / \mathrm{mL}$ VAN $(0.125 \times \mathrm{MIC})$; : FICI 0.625 was obtained at the following concentrations: $32 \mathrm{mcg} / \mathrm{mL}$ COL $(0.125 \times$ MIC $)+16 \mathrm{mcg} / \mathrm{mL}$ VAN $(0.5 \times$ MIC $)$. The fractional inhibitory concentration index (FICI) of each combination was defined as: $\sum$ FIC: FICA + FICB = MICA + B/MICAalone + MICB + A/MICBalone. Synergism was defined as FICI $\leq 0.5$ (S) whereas FICI $>0.5$ but $<4$ were considered as indifferent (I). COL: colistin; MEM: meropenem; RIF: rifampin; VAN: vancomycin; TIG: tigecycline. 
In killing studies, monotherapy showed a significant growth at $24 \mathrm{~h}$ in both colistin-sensitive and colistin-resistant strains. With regard to colistin-based combinations, COL + MEM and COL + TIG were bactericidal and synergistic against both colistin-sensitive and low colistin-resistant strains whereas only the combinations COL + VAN and COL + RIF showed an early and durable bactericidal activity against all the tested strains (including the high colistin-resistant one), with absence of growth at $24 \mathrm{~h}$ (Figure 2A-C). On the other hand, the combination MEM + TIG showed a bactericidal and synergistic activity at $24 \mathrm{~h}$ at the concentrations $1 \times \mathrm{MIC} \mathrm{MEM}+1 \times \mathrm{MIC}$ TIG and $0.5 \times \mathrm{MIC}$ MEM $+0.5 \times$ MIC TIG for the colistin-sensitive and the low-level of colistin-resistance strains whereas no effect at all was observed for the high-level of colistin-resistance strain (Figure 3A-C).

A

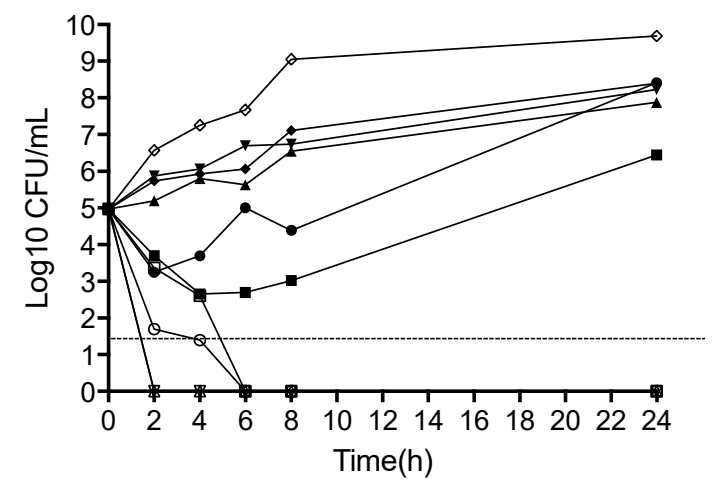

B

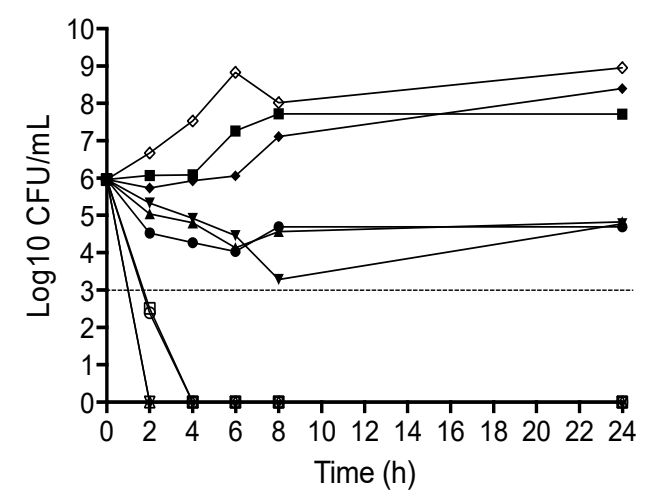

C

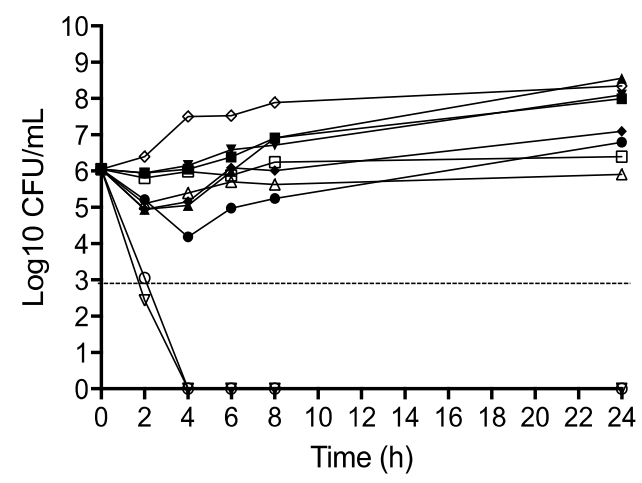

$\rightarrow 1 \times \mathrm{COL}$

$\rightarrow 0.5 \times$ MEM

$\rightarrow 0.5 \times \mathrm{RIF}$

$\rightarrow 0.5 \times$ TIG

$\rightarrow 0.25 \times$ VAN

$\rightarrow 1 \times \mathrm{COL}+0.25 \times \mathrm{VAN}$

$\square 1 \times \mathrm{COL}+0.5 \times \mathrm{MEM}$

$\triangle 1 \times \mathrm{COL}+0.5 \times \mathrm{TIG}$

$\rightarrow 1 \times \mathrm{COL}+0.5 \times \mathrm{RIF}$

$\rightarrow$ GC

- $1 \times \mathrm{COL}$

$\rightarrow 0.5 \times$ MEM

$\leftarrow 0.5 \times \mathrm{RIF}$

$\rightarrow 0.5 \times$ TIG

- $0.25 \times$ VAN

$\rightarrow 1 \times \mathrm{COL}+0.25 \times \mathrm{VAN}$

$\square 1 \times \mathrm{COL}+0.5 \times \mathrm{MEM}$

$\triangle 1 \times \mathrm{COL}+0.5 \times \mathrm{TIG}$

$\rightarrow 1 \times \mathrm{COL}+0.5 \times \mathrm{RIF}$

$\rightarrow$ GC

Figure 2. Killing studies of COL-containing combinations against COL-S (1A), COL-LR (1B) and COL-HR (1C) CR-Ab strains. COL: colistin; VAN: vancomycin; MEM: meropenem; TIG: tigecycline; RIF: rifampin; GC: growth control. Dashed line represents bactericidal activity that occurred at any time point compared to the initial bacterial inoculum [17-20]. 
A

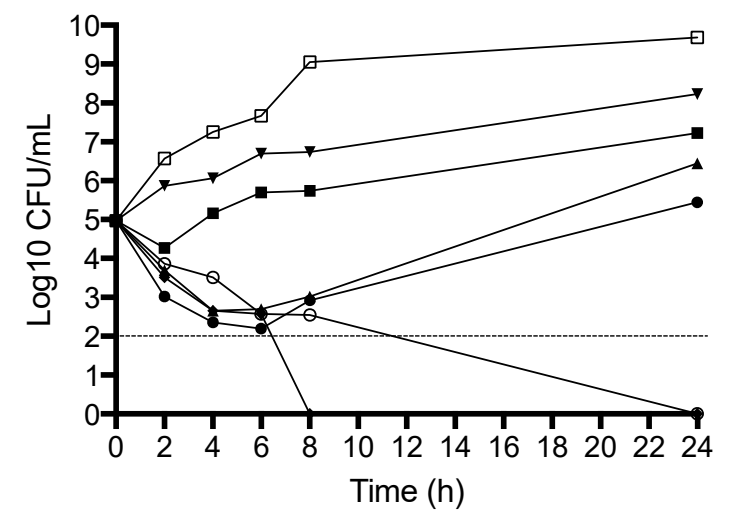

B
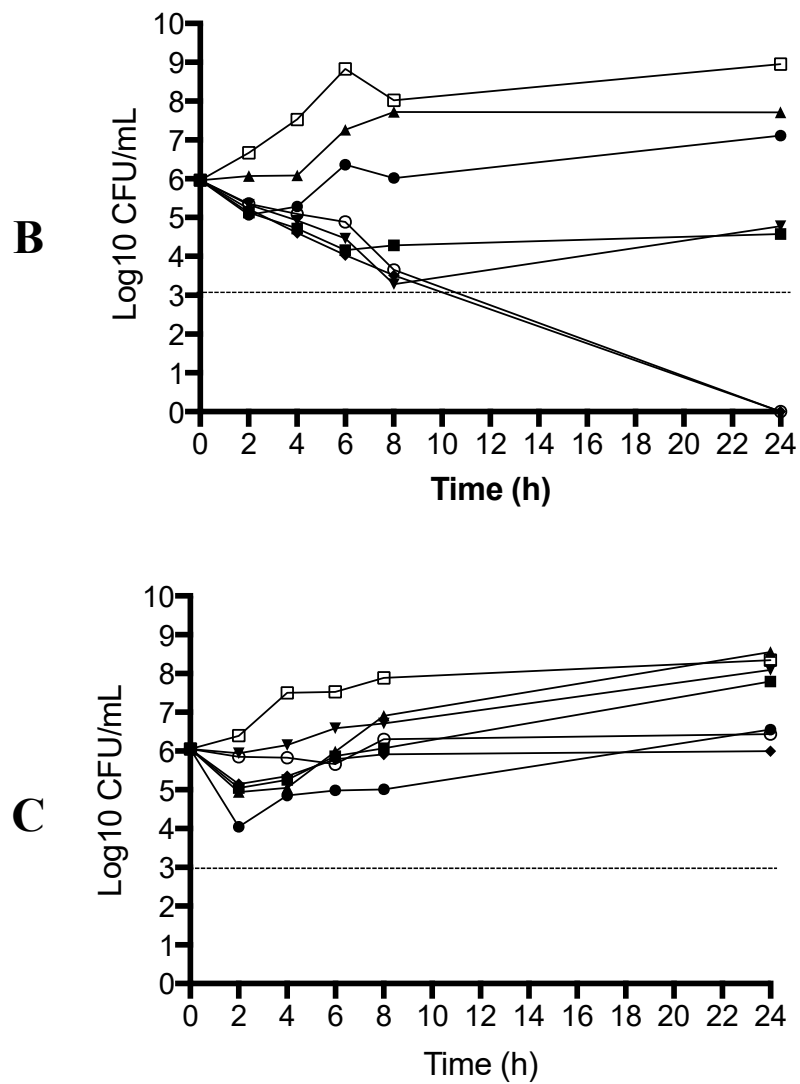

$$
\begin{aligned}
& \rightarrow 1 \times \mathrm{MEM} \\
& \rightarrow 1 \times \mathrm{TIG} \\
& \rightarrow 0.5 \times \mathrm{MEM} \\
& \rightarrow 0.5 \times \mathrm{TIG} \\
& \rightarrow 1 \times \mathrm{MEM}+1 \times \mathrm{TIG} \\
& \rightarrow 0.5 \times \mathrm{MEM}+0.5 \mathrm{TIG} \\
& \rightarrow \mathrm{GC}
\end{aligned}
$$

$\rightarrow 1 \times$ MEM

$-1 \times T I G$

$\rightarrow 0.5 \times \mathrm{MEM}$

$\rightarrow 0.5 \times$ TIG

$\rightarrow 1 \times \mathrm{MEM}+1 \times$ TIG

$-0.5 \times \mathrm{MEM}+0.5 \mathrm{TIG}$

$\rightarrow \mathrm{GC}$

Figure 3. Killing studies of combination without COL against COL-S (2A), COL-LR (2B) and COL-HR (2C) CR-Ab strains. MEM: meropenem; TIG: tigecycline; GC: growth control. Dashed line represents bactericidal activity that occurred at any time point compared to the initial bacterial inoculum.

Regarding a possible relationship between the clonality of isolates and the synergistic effect of different combinations against $\mathrm{CR}-\mathrm{Ab}$, we could say that no synergism was observed for clone $\mathrm{A}$ whereas all the tested combinations were synergic towards clone C. MEM + TIG was mostly in-vitro effective against strains belonging to Clone $\mathrm{B}(3 / 4,75 \%)$ whereas the un-conventional regimen COL + RIF represented the most synergic combination towards clones D and E (2/3 each, 66\%).

\section{Discussion}

Infections caused by $\mathrm{CR}-\mathrm{Ab}$ represent a major challenge for physicians due to the high morbidity and mortality rates [1]. In particular, the growing levels of resistance even to $\mathrm{COL}$, which has been considered as the last resort drug, is particularly worrying due to the already limited therapeutic options available [21]. In this critical scenario, researchers focused their attention on non-conventional antimicrobial combinations (i.e., COL plus antimicrobials active only against Gram-positives) $[11,12]$ 
as well as on non-antimicrobial compounds exhibiting antibacterial properties, such as phages or essential oils [22].

With regard to non-conventional antimicrobial combinations, COL + VAN or COL + RIF gained interest given the high activity in-vitro as well as in animal models $[23,24]$ or even in clinical studies [14,25-27]. While there is concern regarding the possible augmented risk of nephrotoxicity with the combination COL + VAN [26], the association COL + RIF showed a higher microbiological eradication rate than $\mathrm{COL}$ alone, although not influencing the overall mortality compared to COL monotherapy [14]. From a clinical point of view, a recent randomized clinical trial suggested no advantages of $\mathrm{COL}+\mathrm{MEM}$ combination versus COL monotherapy for severe $\mathrm{CR}-\mathrm{Ab}$ infections; however, from a microbiological standpoint, the possibility of evinced resistance to COL during monotherapy is a matter of debate and should be taken into account when considering the optimal regimen (monotherapy versus combination therapy) against CR-Ab [13,28].

In the present study, the aim was to investigate on the in-vitro synergistic and bactericidal activity of different antimicrobial combinations (with and without COL) against strains of CR-Ab isolated from patients with $\mathrm{CR}-\mathrm{Ab}$ infections whose clinical data were also presented. Herein, it has been demonstrated that a combination consisting of COL + VAN or COL + RIF was effective against CR-Ab and might represent a valid therapeutic option for severe infections caused by MDR A. baumannii.

Interestingly, the results of killing studies showed that these combinations were highly effective even in the presence of COL resistance. This finding is in line with previous in-vitro studies that evaluated different combinations against COL-resistant strains and found that COL associated with RIF was the most synergistic one [29-34]. However, the strength of the present study resides on the evaluation of the synergistic effect of the tested combinations, including COL + VAN, by killing studies rather than other methods, thus giving additional information on the kinetic for obtaining the bactericidal and synergistic activity. In fact, it is herein shown that the bactericidal effect of COL + RIF and COL + VAN occurred early, after 2-4 h of incubation, in the absence of bacterial growth for up to $24 \mathrm{~h}$. The rationale of using COL + VAN or RIF relies on COL cell-permeabilizing properties, which might allow associated drug to reach its target at sub-inhibitory concentrations $[10,35,36]$.

Apart from in-vitro results, the clinical experience on the use of these regimens against COL-resistant CR-Ab is still low [15,37]. Thus, in addition to microbiological analyses, in the present report clinical data of patients infected with $\mathrm{CR}-\mathrm{Ab}$ are also presented, including subjects with infections due to COL-resistant strains who received treatment based on COL + VAN or COL + RIF combinations. Herein, the overall mortality was low and did not differ between patients treated with COL + VAN, COL + RIF or other COL-based combinations, with absence of nephrotoxicity in patients receiving $\mathrm{COL}+\mathrm{VAN}$.

A further strength of the study is the investigation of combinations not containing COL, such as MEM + TIG; in fact, this combination could still retain a place in therapy when the use of COL is contraindicated or the risk of COL pharmacokinetic under exposure is a matter of concern [16]. Overall, we found a rate of synergy slightly higher than that found in previous studies by the checkerboard method (36\% vs. $20 \%$ ), which was most prominent in COL sensitive strains [15,38]. In the killing studies, although MEM + TIG was in-vitro effective against COL-sensitive strain, in the presence of low-level of COL resistance the bactericidal activity, although still present, occurred only after $24 \mathrm{~h}$ and, in the presence of high-level of COL resistance, the activity was completely absent. The latter is of particular importance due to main points: i) in the presence of kidney failure or other side effects related to the use of COL, MEM + TIG combination might be considered a better option to treat COL susceptible CR-Ab strains whereas in the presence of high COL resistance this combination may not be effective; ii). The presence of COL resistance represents a condition which might make the isolates more susceptible to COL-containing regimens, probably as a consequence of the alterations in the outer membrane and lipopolysaccharide (LPS) that confer COL resistance, thus raising the vulnerability of $\mathrm{CR}-\mathrm{Ab}$ to these combinations [39]. However, a definite conclusion on the therapeutic role of MEM + 
TIG combination in the presence of COL-resistance cannot be deduced with certainty due to the low number of COL-resistant strains tested.

With regard to the results of PFGE analyses, it was shown that clone B was the most common and exclusively found in one specific ward, whereas clone $C$ belonged to a strain not primarily circulating at SUR hospital. On the other hand, the diffusion of clones A, D and E in wards not related to each other suggests that multiple clones of CR-Ab are simultaneously present in the hospital. In an attempt to evaluate whether the synergistic effect against $\mathrm{CR}-\mathrm{Ab}$ was related to the clonality of the isolates, we found that all the tested combinations did not show the same synergistic effect against different clones of $\mathrm{CR}-\mathrm{Ab}$, with some apparently more effective than others towards a specific clone. However, given the paucity of tested strains, a definite conclusion on the potential role of clonality on determining the synergistic effect of different combinations cannot be drawn [15].

\section{Materials and Methods}

\subsection{Bacterial Strains and Clinical Data Collection}

A series of CR-Ab collected from patients hospitalized at Sapienza University of Rome (SUR) was included in the study. Clinical samples underwent traditional microbiological procedures as for daily practice, with the use of the VITEK-2 (Bio-Merieux, Marcy l'Etoile, France) automated system for identification and antimicrobial susceptibility testing. Until further microbiological analyses, bacteria were stored on cryovial bead preservation system (Microbank; Pro-Lab Diagnostics, Richmond Hill, Ontario, Canada) at $-80^{\circ} \mathrm{C}$. Overnight cultures were then adjusted to a turbidity of $0.5 \mathrm{McFarland}$, corresponding to $\approx 1 \times 10^{8} \mathrm{CFU} / \mathrm{mL}$. For each patient clinical and demographic data were collected. Type of infection was defined in accordance to Centres for Disease Control and Prevention (CDC) guidelines [40]. Clinical cure was defined as resolution of CR-Ab infection. Data collection and microbiological analysis were anonymously and confidentially performed and approved by the Ethics Committee of Policlinico Umberto I of Rome. All experiments were performed in accordance with guidelines and regulations, following the rules of the Declaration of Helsinki.

\subsection{Antimicrobial Agents}

Antimicrobial agents were provided as purified powders by the manufacturer (Sigma Aldrich, Milan, Italy). Stock solutions at different concentrations were prepared in sterile and pyrogen-free $0.9 \%$ saline or water, according to manufacturer's instructions.

\subsection{Molecular Typing by Pulsed-Field Gel Electrophoresis (PFGE)}

The preparation of genomic DNA of CR-Ab isolates was performed as previously described [41]. DNA restriction was done with ApaI enzyme (New England Biolabs, Ipswich, MA, USA) at $25^{\circ} \mathrm{C}$ for $4 \mathrm{~h}$. The gels were run on a CHEF-DRII system (Bio-Rad Laboratories, Hercules, CA, USA) over $20 \mathrm{~h}$ at $14{ }^{\circ} \mathrm{C}$ with 5 to $13 \mathrm{~s}$ of linear ramping at $200 \mathrm{~V}$. It was assumed that a single base mutation in the chromosomal DNA could introduce at most a 3-fragment difference in the restriction pattern [42]; isolates with more than 3 fragment variations were assumed to represent strains with major patterns (assignment of capital letters), while isolates with 1-3 fragment differences were considered subtypes (capital letters with numerical subscripts).

\subsection{Antimicrobial Evaluation}

Minimal inhibitory concentrations (MICs) of MEM, COL, RIF, tigecycline (TIG) and VAN were determined by macro broth dilution (MBD) method in cation-adjusted Mueller Hinton broth (CA-MHB) [43]. Briefly, two-fold serial dilutions of each antimicrobial agent were prepared in 2 $\mathrm{mL}$ CA-MHB in borosilicate glass tubes and incubated for $24 \mathrm{~h}$ at $37^{\circ} \mathrm{C}$. MIC was defined as the lowest concentration of antibiotic that completely inhibited visible growth. In addition, for COL and TIG a gradient strip MIC determination (E-test) was performed. For synergistic activity evaluation, 
checkerboard method of the following antimicrobial combinations was performed: COL + MEM, COL + RIF, COL + TIG, COL + VAN, MEM + TIG. A 96-well microtiter plate containing antibiotic combinations at different concentrations was used to perform checkerboard synergy testing. Wells containing a final inoculum of $\sim 5 \times 10^{5} \mathrm{CFU} / \mathrm{mL}$ were incubated at $37^{\circ} \mathrm{C}$ for $24 \mathrm{~h}$ under static conditions in CA-MHB. The fractional inhibitory concentration index (FICI) of each combination was defined as: $\sum F I C$ : FICA + FICB $=$ MICA + B $/$ MICAalone + MICB + A/MICBalone. Synergism was defined as $\mathrm{FICI} \leq 0.5$ whereas FICI $>0.5$ but $<4$ were considered as indifferent or non-antagonistic [44]. In addition, killing studies of different antimicrobial combinations were performed on 3 representative strains, according to COL sensitivity [one strain with full COL susceptibility (COL-S); one strain with low-level of COL resistance (COL- LR); one strain with high-level of COL resistance (COL-HR)]. COL susceptibility or resistance was defined in accordance to the European Committee on Antimicrobial Susceptibility Testing (EUCAST) clinical breakpoint $[18,19]$. The following concentrations were used for killing tests: $1 \times$ MIC COL, $0.5 \times$ MIC RIF, $0.5 \times$ MIC MEM, $0.5 \times$ MIC TIG, VAN, $1 \times$ MIC COL + VAN, $1 \times$ MIC COL $+0.5 \times$ MIC TIG, $1 \times$ MIC COL $+0.5 \times$ MIC RIF, $1 \times$ MIC COL +0.5 $\times$ MIC MEM, $0.5 \times$ MIC MEM $+0.5 \times$ MIC TIG, $1 \times$ MIC MEM $+1 \times$ MIC TIG. The tested VAN concentration was fixed at $16 \mathrm{mg} / \mathrm{L}$, which reflects the serum achievable concentration during VAN therapy [25]. Bactericidal activity was defined as $\geq 99.9 \%$ (i.e., $\geq 3-\log 10 \mathrm{CFU} / \mathrm{mL}$ ) reduction of the initial bacterial count at each time point. Synergy was defined as a $\geq 100$-fold decrease in CFU / mL between the combination and its most active constituent at the same concentration after $24 \mathrm{~h}$, with the number of surviving organisms in the presence of the combination $\geq 100$-fold CFU $/ \mathrm{mL}$ below the starting inoculum. The MIC values used for synergy testing were those obtained throughout MBD. All in-vitro experiments were performed in triplicate.

\section{Conclusions}

In conclusion, it is herein demonstrated that combinations of COL with VAN or RIF were highly synergic and rapidly bactericidal against $\mathrm{CR}-\mathrm{Ab}$, including COL-resistant strains and that, in the presence of kidney failure or other side effects related to the use of COL, MEM + TIG might be considered a reasonable approach to treat mainly COL susceptible CR-Ab strains.

Author Contributions: Conceptualization, A.O. and R.R.; methodology, A.O., S.G., C.M.; formal analysis, M.D.A., S.G., C.M.; data curation, A.O., R.R.; writing—original draft preparation, A.O.; writing-review and editing, R.R., C.M.M., V.V.

Funding: This work was supported by a grant of Sapienza University of Rome, 2014 (Number: C26N14S7RB).

Acknowledgments: The authors thank the technician staff (Silvia Costantini, Federica Di Timoteo) for their contribution in collecting laboratory samples. We are also very grateful to Virginia Filacchione for the English revision of the manuscript.

Conflicts of Interest: The authors declare no conflict of interest.

\section{References}

1. Munoz-Price, L.S.; Poirel, L.; Bonomo, R.A.; Schwaber, M.J.; Daikos, G.L.; Cormican, M.; Cornaglia, G.; Garau, J.; Gniadkowski, M.; Hayden, M.K.; et al. Clinical epidemiology of the global expansion of Klebsiella pneumoniae carbapenemases. Lancet Infect. Dis. 2013, 13, 785-796. [CrossRef]

2. Maragakis, L.L.; Perl, T.M. Acinetobacter baumannii: Epidemiology, antimicrobial resistance, and treatment options. Clin. Infect. Dis. 2008, 46, 1254-1263. [CrossRef] [PubMed]

3. Harding, C.M.; Hennon, S.W.; Feldman, M.F. Uncovering the mechanisms of Acinetobacter baumannii virulence. Nat. Rev. Microbiol. 2018, 16, 91-102. [CrossRef] [PubMed]

4. Russo, A.; Giuliano, S.; Ceccarelli, G.; Alessandri, F.; Giordano, A.; Brunetti, G.; Venditti, M. Comparison of Septic Shock Due to Multidrug-Resistant Acinetobacter baumannii or Klebsiella pneumoniae Carbapenemase-Producing K. pneumoniae in Intensive Care Unit Patients. Antimicrob. Agents Chemother. 2018, 62, e02562-17. [CrossRef] [PubMed] 
5. Vila, J.; Pachon, J. Therapeutic options for Acinetobacter baumannii infections: An update. Expert Opin. Pharmacother. 2012, 13, 2319-2336. [CrossRef] [PubMed]

6. Asif, M.; Alvi, I.A.; Rehman, S.U. Insight into Acinetobacter baumannii: Pathogenesis, global resistance, mechanisms of resistance, treatment options, and alternative modalities. Infect. Drug Resist. 2018, 11, 1249-1260. [CrossRef] [PubMed]

7. Falagas, M.E.; Mavroudis, A.D.; Vardakas, K.Z. The antibiotic pipeline for multi-drug resistant gram negative bacteria: What can we expect? Expert Rev. Anti Infect. Ther. 2016, 14, 747-763. [CrossRef] [PubMed]

8. Choi, J.J.; McCarthy, M.W. Cefiderocol: A novel siderophore cephalosporin. Expert Opin. Investig. Drugs 2018, 27, 193-197. [CrossRef] [PubMed]

9. Lertsrisatit, Y.; Santimaleeworagun, W.; Thunyaharn, S.; Traipattanakul, J. In vitro activity of colistin monoand combination therapy against colistin-resistant Acinetobacter baumannii, mechanism of resistance, and clinical outcomes of patients infected with colistin-resistant A. baumannii at a Thai university hospital. Infect. Drug Resist. 2017, 10, 437-443. [CrossRef] [PubMed]

10. Gordon, N.C.; Png, K.; Wareham, D.W. Potent synergy and sustained bactericidal activity of a vancomycin-colistin combination versus multidrug-resistant strains of Acinetobacter baumannii. Antimicrob. Agents Chemother. 2010, 54, 5316-5322. [CrossRef] [PubMed]

11. Motaouakkil, S.; Charra, B.; Hachimi, A.; Nejmi, H.; Benslama, A.; Elmdaghri, N.; Belabbes, H.; Benbachir, M. Colistin and rifampicin in the treatment of nosocomial infections from multiresistant Acinetobacter baumannii. J. Infect. 2006, 53, 274-278. [CrossRef] [PubMed]

12. O'Hara, J.A.; Ambe, L.A.; Casella, L.G.; Townsend, B.M.; Pelletier, M.R.; Ernst, R.K.; Shanks, R.M.; Doi, Y. Activities of vancomycin-containing regimens against colistin-resistant Acinetobacter baumannii clinical strains. Antimicrob. Agents Chemother. 2013, 57, 2103-2108. [CrossRef] [PubMed]

13. Paul, M.; Daikos, G.L.; Durante-Mangoni, E.; Yahav, D.; Carmeli, Y.; Benattar, Y.D.; Skiada, A.; Andini, R.; Eliakim-Raz, N.; Nutman, A.; et al. Colistin alone versus colistin plus meropenem for treatment of severe infections caused by carbapenem-resistant Gram-negative bacteria: An open-label, randomised controlled trial. Lancet Infect. Dis. 2018, 18, 391-400. [CrossRef]

14. Durante-Mangoni, E.; Signoriello, G.; Andini, R.; Mattei, A.; De Cristoforo, M.; Murino, P.; Bassetti, M.; Malacarne, P.; Petrosillo, N.; Galdieri, N.; et al. Colistin and rifampicin compared with colistin alone for the treatment of serious infections due to extensively drug-resistant Acinetobacter baumannii: A multicenter, randomized clinical trial. Clin. Infect. Dis. 2013, 57, 349-358. [CrossRef] [PubMed]

15. Leite, G.C.; Oliveira, M.S.; Perdigao-Neto, L.V.; Rocha, C.K.; Guimaraes, T.; Rizek, C.; Levin, A.S.; Costa, S.F. Antimicrobial Combinations against Pan-Resistant Acinetobacter baumannii Isolates with Different Resistance Mechanisms. PLoS ONE 2016, 11, e0151270. [CrossRef] [PubMed]

16. Biswas, S.; Brunel, J.M.; Dubus, J.C.; Reynaud-Gaubert, M.; Rolain, J.M. Colistin: an update on the antibiotic of the 21st century. Expert Rev. Anti Infect. Ther. 2012, 10, 917-934. [CrossRef] [PubMed]

17. Horan, T.C.; Andrus, M.; Dudeck, M.A. CDC/NHSN surveillance definition of health care-associated infection and criteria for specific types of infections in the acute care setting. Am. J. Infect. Control 2008, 36, 309-332. [CrossRef] [PubMed]

18. Villari, P.; Iacuzio, L.; Vozzella, E.A.; Bosco, U. Unusual genetic heterogeneity of Acinetobacter baumannii isolates in a university hospital in Italy. Am. J. Infect. Control 1999, 27, 247-253. [CrossRef] [PubMed]

19. Tenover, F.C.; Arbeit, R.D.; Goering, R.V.; Mickelsen, P.A.; Murray, B.E.; Persing, D.H.; Swaminathan, B. Interpreting chromosomal DNA restriction patterns produced by pulsed-field gel electrophoresis: Criteria for bacterial strain typing. J. Clin. Microbiol. 1995, 33, 2233-2239. [PubMed]

20. CLSI. M07-A9; Methods for Dilution Antimicrobial Susceptibility Tests for Bacteria That Grow Aerobically, 9th ed.; Wayne, P.A., Ed.; Clinical and Laboratory Standards Institute: Wayne, PA, USA, 2012; Volume 32.

21. Odds, F.C. Synergy, antagonism, and what the chequerboard puts between them. J. Antimicrob. Chemother. 2003, 52, 1. [CrossRef] [PubMed]

22. Bengtsson, S.; Bjelkenbrant, C.; Kahlmeter, G. Validation of EUCAST zone diameter breakpoints against reference broth microdilution. Clin. Microbiol. Infect. 2014, 20, O353-O360. [CrossRef] [PubMed]

23. Brown, D.; Canton, R.; Dubreuil, L.; Gatermann, S.; Giske, C.; MacGowan, A.; Martinez-Martinez, L.; Mouton, J.; Skov, R.; Steinbakk, M.; et al. Widespread implementation of EUCAST breakpoints for antibacterial susceptibility testing in Europe. Euro Surveill. 2015, 20, 21008. [CrossRef] [PubMed] 
24. Ceccarelli, G.; Oliva, A.; d'Ettorre, G.; D'Abramo, A.; Caresta, E.; Barbara, C.S.; Mascellino, M.T.; Papoff, P.; Moretti, C.; Vullo, V.; et al. The role of vancomycin in addition with colistin and meropenem against colistin-sensitive multidrug resistant Acinetobacter baumannii causing severe infections in a Paediatric Intensive Care Unit. BMC Infect. Dis. 2015, 15, 393. [CrossRef] [PubMed]

25. Humphries, R.M.; Ambler, J.; Mitchell, S.L.; Castanheira, M.; Dingle, T.; Hindler, J.A.; Koeth, L.; Sei, K.; Development, C.M.; Standardization Working Group of the Subcommittee on Antimicrobial Susceptibility Testing. CLSI Methods Development and Standardization Working Group Best Practices for Evaluation of Antimicrobial Susceptibility Tests. J. Clin. Microbiol. 2018, 56, e01934-17. [CrossRef] [PubMed]

26. Brown, D.F.; Wootton, M.; Howe, R.A. Antimicrobial susceptibility testing breakpoints and methods from BSAC to EUCAST. J. Antimicrob. Chemother. 2016, 71, 3-5. [CrossRef] [PubMed]

27. Qureshi, Z.A.; Hittle, L.E.; O’Hara, J.A.; Rivera, J.I.; Syed, A.; Shields, R.K.; Pasculle, A.W.; Ernst, R.K.; Doi, Y. Colistin-resistant Acinetobacter baumannii: Beyond carbapenem resistance. Clin. Infect. Dis. 2015, 60, 1295-1303. [CrossRef] [PubMed]

28. Oliva, A.; Costantini, S.; De Angelis, M.; Garzoli, S.; Bozovic, M.; Mascellino, M.T.; Vullo, V.; Ragno, R. High Potency of Melaleuca alternifolia Essential Oil against Multi-Drug Resistant Gram-Negative Bacteria and Methicillin-Resistant Staphylococcus aureus. Molecules 2018, 23, 2584. [CrossRef] [PubMed]

29. Fan, B.; Guan, J.; Wang, X.; Cong, Y. Activity of Colistin in Combination with Meropenem, Tigecycline, Fosfomycin, Fusidic Acid, Rifampin or Sulbactam against Extensively Drug-Resistant Acinetobacter baumannii in a Murine Thigh-Infection Model. PLoS ONE 2016, 11, e0157757. [CrossRef] [PubMed]

30. Hornsey, M.; Wareham, D.W. In vivo efficacy of glycopeptide-colistin combination therapies in a Galleria mellonella model of Acinetobacter baumannii infection. Antimicrob. Agents Chemother. 2011, 55, 3534-3537. [CrossRef] [PubMed]

31. Garnacho-Montero, J.; Amaya-Villar, R.; Gutierrez-Pizarraya, A.; Espejo-Gutierrez de Tena, E.; Artero-Gonzalez, M.L.; Corcia-Palomo, Y.; Bautista-Paloma, J. Clinical efficacy and safety of the combination of colistin plus vancomycin for the treatment of severe infections caused by carbapenem-resistant Acinetobacter baumannii. Chemotherapy 2013, 59, 225-231. [CrossRef] [PubMed]

32. Petrosillo, N.; Giannella, M.; Antonelli, M.; Antonini, M.; Barsic, B.; Belancic, L.; Inkaya, A.C.; De Pascale, G.; Grilli, E.; Tumbarello, M.; et al. Clinical experience of colistin-glycopeptide combination in critically ill patients infected with Gram-negative bacteria. Antimicrob. Agents Chemother. 2014, 58, 851-858. [CrossRef] [PubMed]

33. Pogue, J.M.; Kaye, K.S. Is there really no benefit to combination therapy with colistin? Expert Rev. Anti Infect. Ther. 2013, 11, 881-884. [CrossRef] [PubMed]

34. Hong, D.J.; Kim, J.O.; Lee, H.; Yoon, E.J.; Jeong, S.H.; Yong, D.; Lee, K. In vitro antimicrobial synergy of colistin with rifampicin and carbapenems against colistin-resistant Acinetobacter baumannii clinical isolates. Diagn. Microbiol. Infect. Dis. 2016, 86, 184-189. [CrossRef] [PubMed]

35. Lee, H.J.; Bergen, P.J.; Bulitta, J.B.; Tsuji, B.; Forrest, A.; Nation, R.L.; Li, J. Synergistic activity of colistin and rifampin combination against multidrug-resistant Acinetobacter baumannii in an in vitro pharmacokinetic/pharmacodynamic model. Antimicrob. Agents Chemother. 2013, 57, 3738-3745. [CrossRef] [PubMed]

36. Tascini, C.; Tagliaferri, E.; Giani, T.; Leonildi, A.; Flammini, S.; Casini, B.; Lewis, R.; Ferranti, S.; Rossolini, G.M.; Menichetti, F. Synergistic activity of colistin plus rifampin against colistin-resistant KPC-producing Klebsiella pneumoniae. Antimicrob. Agents Chemother. 2013, 57, 3990-3993. [CrossRef] [PubMed]

37. Wei, W.; Yang, H.; Liu, Y.; Ye, Y.; Li, J. In vitro synergy of colistin combinations against extensively drug-resistant Acinetobacter baumannii producing OXA-23 carbapenemase. J. Chemother. 2016, 28, 159-163. [CrossRef] [PubMed]

38. Brennan-Krohn, T.; Pironti, A.; Kirby, J.E. Synergistic Activity of Colistin-Containing Combinations against Colistin-Resistant Enterobacteriaceae. Antimicrob. Agents Chemother. 2018, 62, e00873-18. [CrossRef] [PubMed]

39. MacNair, C.R.; Stokes, J.M.; Carfrae, L.A.; Fiebig-Comyn, A.A.; Coombes, B.K.; Mulvey, M.R.; Brown, E.D. Overcoming mcr-1 mediated colistin resistance with colistin in combination with other antibiotics. Nat. Commun. 2018, 9, 458. [CrossRef] [PubMed] 
40. Vaara, M. Agents that increase the permeability of the outer membrane. Microbiol. Rev. 1992, 56, $395-411$. [PubMed]

41. Vidaillac, C.; Benichou, L.; Duval, R.E. In vitro synergy of colistin combinations against colistin-resistant Acinetobacter baumannii, Pseudomonas aeruginosa, and Klebsiella pneumoniae isolates. Antimicrob. Agents Chemother. 2012, 56, 4856-4861. [CrossRef] [PubMed]

42. Oliva, A.; Cipolla, A.; Vullo, V.; Venditti, M.; Mastroianni, C.M.; Falcone, M. Clinical and in vitro efficacy of colistin plus vancomycin and rifampin against colistin-resistant Acinetobacter baumannii causing ventilator-associated pneumonia. New Microbiol. 2017, 40, 205-207. [PubMed]

43. Jiang, Z.; He, X.; Li, J. Synergy effect of meropenem-based combinations against Acinetobacter baumannii: A systematic review and meta-analysis. Infect. Drug Resist. 2018, 11, 1083-1095. [CrossRef] [PubMed]

44. Al-Shaer, M.; Nazer, L.H.; Kherallah, M. Rifampicin as adjunct to colistin therapy in the treatment of multidrug-resistant Acinetobacter baumannii. Ann. Pharmacother. 2014, 48, 766-771. [CrossRef] [PubMed]

Sample Availability: Samples of the compounds are not available from the authors. 